\title{
Direct observations of nucleation in a nondilute multicomponent alloy
}

\author{
Chantal K. Sudbrack,, , R Ronald D. Noebe, ${ }^{2}$ and David N. Seidman ${ }^{1, \dagger}$ \\ 1 Northwestern University, Department of Materials Science and Engineering, Evanston, IL 60208 USA \\ ${ }^{2}$ NASA Glenn Research Center, Cleveland, OH 44135 USA
}

(Dated: November 19, 2018)

\begin{abstract}
The chemical pathways leading to $\gamma^{\prime}\left(\mathrm{L}_{2}\right)$-nucleation from nondilute $\mathrm{Ni}-5.2 \mathrm{Al}-14.2 \mathrm{Cr}$ at.\%, $\gamma$ (f.c.c.), at $873 \mathrm{~K}$ are followed with radial distribution functions and isoconcentration surface analyses of direct-space atom-probe tomographic images. Although $\mathrm{Cr}$ atoms initially are randomly distributed, a distribution of congruent $\mathrm{Ni}_{3} \mathrm{Al}$ short-range order domains ( $\mathrm{SRO}$ ), $<R>\cong 0.6 \mathrm{~nm}$, results from $\mathrm{Al}$ diffusion during quenching. Domain site occupancy develops as their number density increases leading to Al-rich phase separation by $\gamma^{\prime}$-nucleation, $<R>=0.75 \mathrm{~nm}$, after SRO occurs.
\end{abstract}

PACS numbers: $64.75 .+\mathrm{g}, 81.30 . \mathrm{Hd}, 68.37 . \mathrm{Vj}, 61.18 .-\mathrm{j}$

A fundamental aspect of any phase transition involves the nucleation of stable nuclei. The theoretical nucleation literature is vast, e.g. 1, 2, 3], whereas direct experimental observations of sub-nano to nanoscale nuclei are miniscule [4], controversial [5], and limited to binary systems 4]. Experimental studies to date have measured the kinetics of nuclei with radii, $R,>2 \mathrm{~nm}$ [4], with no knowledge of the chemical pathways associated with nucleation. With atom-probe tomography (APT), it is possible to characterize both the spatial extent and compositions of nuclei as small as $0.45 \mathrm{~nm}[\underline{6}]$ with direct-space atomic reconstructions of small volumes of material (typically $2 \times 10^{4} \mathrm{~nm}^{3}$ containing $10^{6}$ atoms). We demonstrate herein that APT can also follow the chemical pathways prior to (i.e. ordering and clustering) and during nucleation of $\mathrm{Ni}_{3}\left(\mathrm{Al}_{x} \mathrm{Cr}_{1-x}\right) \gamma^{\prime}$-phase $\left(\mathrm{L}_{2}\right)$ in a moderately supersaturated $\gamma$-matrix (f.c.c.) with isoconcentration surfaces [7] and radial distribution function (RDF) [8] analyses.

High purity elements were induction melted in Ar and then chill cast in a copper mold (diam. $=19 \mathrm{~mm})$. Cast ingots are homogenized in $\mathrm{Ar}$ at $1573 \mathrm{~K}$ for $24 \mathrm{~h}$ and then $1123 \mathrm{~K}$ for $3 \mathrm{~h}$; subsequently $10 \mathrm{~mm}$ thick waterquenched sections are aged at $873 \mathrm{~K}(120 \mathrm{~s}$ to $1024 \mathrm{~h})$. Aged specimens are electropolished to produce sharplypointed needle-shaped specimens for APT [6]. The solute distributions in $3 \mathrm{~nm}$ thick partial APT volumes (Fig. 11) provide direct visual evidence of phase separation (i.e. $\gamma^{\prime}$-nucleation) and demonstrates that atoms are positioned with sub-nanometer resolution with APT. Employing 9 at.\% $\mathrm{Al}$ isoconcentration surface analyses, spheroidal Al-rich regions with $R$ as small as $0.45 \mathrm{~nm}$, 20 detected atoms (ion detection efficiency of $60 \%$ ), are first observed within APT images at $600 \mathrm{~s}$. Along the [001]-direction in $\mathrm{L}_{2} \mathrm{Ni}_{3}\left(\mathrm{Al}_{x} \mathrm{Cr}_{1-x}\right)$, nearly pure Ni-rich planes alternate with solute-rich planes. This alternation is captured within the delineated Al-rich regions, i.e. the Al-rich $\{002\}$-superlattice planes $\left(2 d_{200}=0.356\right.$ $\mathrm{nm}$ ) are resolved, thus providing further evidence that these regions are $\gamma^{\prime}$-precipitates. Between 600 and $900 \mathrm{~s}$, $\gamma^{\prime}$-number density, $N_{v}$, increases sharply from $3.6 \times 10^{23}$ to $2.1 \times 10^{24} \mathrm{~m}^{-3}$ at a constant $<R>$ of $0.75 \mathrm{~nm}$, implying that the transformation is undergoing quasi-steadystate nucleation. Their average composition, Ni-18.3 \pm 0.9 Al-9.3 $\pm 0.7 \mathrm{Cr}$ at.\%, estimates a lower-bound for the $\gamma$ 'critical nucleus. We find the $\chi^{2}$-test $[\underline{9}]$ is inadequate to evaluate non-randomness of the $\mathrm{Al}$ and $\mathrm{Cr}$ distributions obtained with APT. Alternatively, RDF analyses offer a higher degree of spatial sensitivity and resolve lattice site-specific information, thereby allowing the chemical pathways associated with $\gamma^{\prime}$-nucleation $(t \leq 600 \mathrm{~s})$ to be followed in direct-space for the first-time.

An RDF at a given radial distance, $r$, is defined as the average concentration distribution of component $i$ around a given solute species, $\mathrm{X},\left\langle C_{i}^{X}(r)>\right.$, normalized to the overall concentration of $i$ atoms, $C_{i}^{o}$, in the sampled volume:

$$
R D F=\frac{<C_{i}^{X}(r)>}{C_{i}^{o}}=\frac{1}{C_{i}^{o}} \sum_{k=1}^{N_{X}} \frac{N_{i}^{k}(r)}{N_{T O T}^{k}(r)}
$$

where $N_{i}^{k}(r)$ is the number of $i$ atoms in a radial shell around the $k^{t h} \mathrm{X}$ atom that is centered at $r, N_{T O T}^{k}(r)$ is the total number of atoms in this shell, and $N_{X}$ is the number of $\mathrm{X}$ atoms in this volume. The average concentration distributions around a solute species in $0.01 \mathrm{~nm}$ thick shells are smoothed with a weighted moving average using a Gaussian-like spline-function [10], with a $0.04 \mathrm{~nm}$ full-width half-maximum, where $\left\langle N_{T O T}(r)\right\rangle$ increases quadratically with $r$ and the total volume sampled for a given shell is proportional to X. Only the RDFs for $r$ $\geq 0.2 \mathrm{~nm}$ are presented as the physical interpretation at smaller $r$ is difficult due to possible ion trajectory effects. $\mathrm{RDF}$ values of unity describe perfectly random distributions, while values that differ describe clustering or ordering. The absolute magnitude of these processes can be compared with the RDF's amplitude, $A=[\operatorname{RDF}(r)-$ 1]; where $A>0$ indicates a greater concentration than the overall concentration (positive correlation) and $A<$ 0 implies a smaller one (negative correlation).

To evaluate an RDF's shape and magnitude when analyzing APT data, which is affected by the APT's po- 


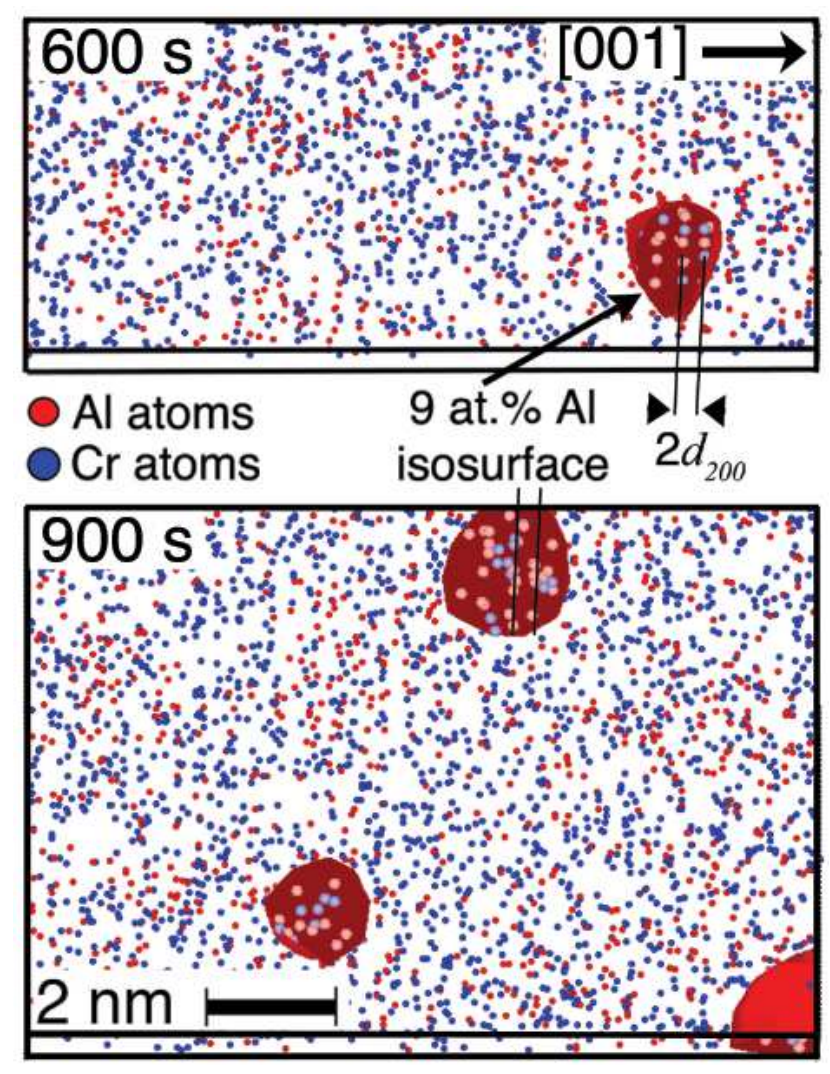

FIG. 1: Within Ni-5.2 Al-14.2 Cr at.\% APT reconstructed volumes, Al-rich $\gamma^{\prime}$-precipitates are first detected with 9 at.\% $\mathrm{Al}$ isoconcentration surfaces after aging for $600 \mathrm{~s}(t o p)$ at $873 \mathrm{~K}$. Between $600 \mathrm{~s}($ top $)$ and $900 \mathrm{~s}$ (bottom), the $<R>$ remains constant $(0.75 \mathrm{~nm}, \sim 150$ atoms $)$; however, a sharp increase in number density from $3.6 \times 10^{23} \mathrm{~m}^{-3}$ to $2.1 \times 10^{24}$ $\mathrm{m}^{-3}$ is observed. The $\mathrm{Al}$ and $\mathrm{Cr}$ atoms in these $3 \mathrm{~nm}$ thick partial volumes, displayed in red and blue, are enlarged in the $\gamma^{\prime}$-precipitates to emphasize the resolved $\{002\}$ superlattice planes perpendicular to the [001] analysis direction, where the average $\gamma^{\prime}$-composition is $\mathrm{Ni}-18.3 \pm 0.9 \mathrm{Al}-9.3 \pm 0.7 \mathrm{Cr}$ at.\%.

sitional uncertainty, we first consider a well-defined system; a nearly-pure $\mathrm{Ni}_{3} \mathrm{Al}\left(\mathrm{L}_{2}\right)$ alloy 11 that exhibits almost perfect long-range order (LRO) [12] and compare it to the expected values for a theoretical $\mathrm{Ni}_{3} \mathrm{Al}$ alloy with $\mathrm{LRO}=1$, Fig. 2. In the theoretical alloy, the $\mathrm{Al}-\mathrm{Al}$ profile (Eq. 1) alternates discretely as a series of deltafunctions between $A=-1$ and $A=3$ at successive nearest neighbor (NN) distances (grey vertical lines in Fig. 2), while the Al- $N i$ profile alternates between $1 / 3$ and -1 (not displayed). Because mass conservation obtains, one RDF in a binary alloy is sufficient to describe the LRO behavior; therefore, only the $\mathrm{Al}-A l$ profiles are presented. The random-state, $\mathrm{RDF}=1$, is denoted by a dashed horizontal line. Compared to the theoretical profile (right-hand ordinate), the experimental profile (left-hand ordinate) is damped (ca. nine times smaller in $A$ than the theoretical value at the $1^{\text {st }} \mathrm{NN}$ distance) and does not follow exactly the theoretical positive and negative alternation.

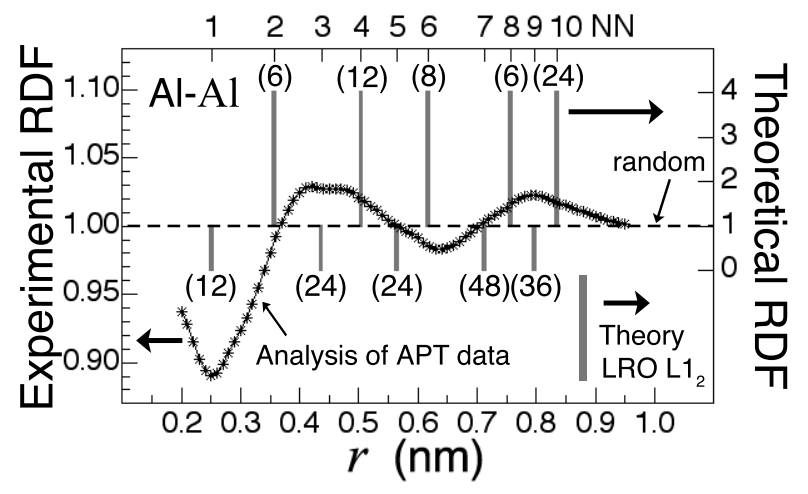

FIG. 2: Al- $A l$ RDFs (Eq. 1) vs. radial distance, $r$, out to the $10^{t h}$ nearest neighbor (NN) distance for a nearly stoichiometric $\mathrm{Ni}_{3} \mathrm{Al}\left(\mathrm{L}_{2}\right)$ alloy 11$]$ analyzed by $\mathrm{APT}$ and a theoretical alloy assuming that the $\mathrm{LRO}=1$. The number of $\mathrm{L}_{2} \mathrm{NNs}$ at corresponding distances is indicated in parentheses. $\mathrm{RDF}=$ 1 (random solid solution) is indicated by a horizontal dashed line.

The experimental values of the $\mathrm{Al}-\mathrm{Al}$ and $\mathrm{Al}-\mathrm{Ni} \mathrm{RDFs}$ are strongest at the $1^{s t} \mathrm{NN}$ distances than at larger $r$. These features of the RDF result from contributions of adjacent NNs to the measured concentrations and is a function of the number of NNs (parenthetical values in Fig. 22) and their weighted contribution (proportional to theoretical $A$ values). Note the measured RDFs oscillate over the full range of $r, r \leq 1 \mathrm{~nm}$, which is a distinguishing feature of LRO in an APT RDF. The differences with theory are understood by considering the value for a particular NN. As the RDF in Eq. 1 is not normalized to the coordination number, $Z$ (as $Z$ assignment is not straightforward due to positional uncertainty), a greater number of NNs contribute more to the measured RDF than fewer with the same weight. At the $3^{\text {rd }} \mathrm{NN}$ distance, although theory predicts a negative correlation, the observed positive correlation results from the positive contributions from the six adjacent $2^{\text {nd }} \mathrm{NNs}$ and $124^{\text {th }} \mathrm{NNs}$, each with a larger weighting factor $(A=3)$ than the $243^{\text {rd }}$ NNs $(A=-1)$. Nevertheless the experimental results in Fig. 2 are indicative of LRO and act as a reference state for what follows. If the distributions of reconstructed atom positions about each NN distance are known, it would be possible to obtain a deconvoluted $\mathrm{RDF}$, allowing pair-wise interaction energies to be determined experimentally in multicomponent systems.

Figure 3 presents experimentally determined Al- and Cr-centered RDFs for the as-quenched (AQ) state (open circles) and after $120 \mathrm{~s}$ (solid diamonds) of aging. The comparison to the random state (dashed horizontal line) checks the homogeneity of the post-quenching state. The nearly flat $\mathrm{Cr}-\mathrm{Cr}$ and $\mathrm{Cr}-\mathrm{Ni} \mathrm{AQ}$ profiles indicate that the quench-rate is sufficient to produce a random $\mathrm{Cr}$ distribution relative to other $\mathrm{Cr}$ and $\mathrm{Ni}$ atoms. The initial quench rate is, however, insufficient to avoid diffusion of 
$\mathrm{Al}$ away from $\mathrm{Cr}$, as $\mathrm{Al}$ diffuses considerably faster than $\mathrm{Cr}$ does in Ni-Al-Cr. The Al- $\mathrm{Al}$ and Al-Ni AQ RDFs' deviations from the random state are comparable to the ones for the LRO alloy, Fig. 22. Unlike the LRO alloy they occur over a shorter range, $r \leq 0.6 \mathrm{~nm}$. At the $1^{\text {st }} \mathrm{NN}$ distance, the $\mathrm{Al}-\mathrm{Al}$ and $\mathrm{Al}-\mathrm{Ni}$ correlations are negative and positive, respectively, and the Al- $A l$ RDF's oscillations are coupled with oscillations in the $\mathrm{Al}-\mathrm{Ni} \mathrm{RDF}$ that are opposite in sign, which is consistent with $\mathrm{L} 1_{2}$ ordering. These trends establish the presence of quenched-in $\mathrm{L} 1_{2}$ SRO domains with $\langle R>\cong 0.6 \mathrm{~nm}$. After $120 \mathrm{~s}$, an increase in the $\mathrm{Al}-\mathrm{Al}$ and $\mathrm{Al}-\mathrm{Ni}$ RDFs' intensities, which also extends $0.6 \mathrm{~nm}$, indicates the SRO in solution increases. It follows that the SRO domains' $<R>$ remains ca. constant as their $N_{v}$ increases concomitantly. Since the solute concentrations are nondilute, the volumes are reasonably well-sampled, yielding $\mathrm{Al}-\mathrm{Cr}$ and $\mathrm{Cr}-\mathrm{Al}$ profiles that are very similar. These RDFs exhibit a negative spatial correlation over the distances analyzed, $r<1 \mathrm{~nm}$, thereby establishing that the $\gamma^{\prime}$-SRO regions are $\mathrm{Cr}$ depleted. In concert with more developed SRO at $120 \mathrm{~s}$, the intensity of the Al- $C r$ RDF at the $1^{s t} \mathrm{NN}$ distance is strongly negative, which suggests that $\mathrm{Cr}$ atoms do not prefer to be $\mathrm{NNs}$ to $\mathrm{Al}$ and that they occupy the $\mathrm{Al}$ sites within the $\mathrm{Ni}_{3}\left(\mathrm{Al}_{x} \mathrm{Cr}_{1-x}\right)$ SRO domains. A slight $\mathrm{Ni}-\mathrm{Cr}$ ordering tendency, either within Al-rich domains or in the $\gamma$-matrix, is observed at $120 \mathrm{~s}$.

Since the RDF is applied to total volumes, as the alloy decomposes $(t \geq 120 \mathrm{~s}$, Fig. 道) the RDF becomes a convolution of the ordering and clustering processes in both $\gamma$ - and $\gamma^{\prime}$-phases, where cumulative shifts in the RDF away from unity (Eq. 1) correspond to elemental partitioning associated with $\gamma^{\prime}$-nucleation. Isoconcentration surfaces (Fig. 1) first detect a small volume fraction $(0.11 \pm 0.04 \%)$ of $\gamma^{\prime}$-nuclei $(R \geq 0.45 \mathrm{~nm})$ that are highly enriched in $\mathrm{Al}(19.1 \pm 1.7 \%)$ at $600 \mathrm{~s}$. Correspondingly the $\mathrm{Al}-A l$ RDF shifts strongly. In comparison, the shift at $300 \mathrm{~s}$ is slight (no appreciable phase separation) and no $\gamma^{\prime}$-nuclei are detected with isoconcentration surfaces, establishing that the onset of $\gamma$ '-phase separation occurs between 300 and $600 \mathrm{~s}$. For $t=0$ (Fig. 3), 120 and 300 $\mathrm{s}$ (Fig. (4), since the Al- $\mathrm{Al}$ profiles oscillate about unity, the SRO domains observed prior to phase separation are congruently ordered, i.e., their compositions do not deviate from the overall concentration. The strong anti-site $\mathrm{Cr}$ preference for $\mathrm{Al}$ in the domains at $120 \mathrm{~s}$ diminishes with further aging, where a decrease in negative correlation at the $1^{\text {st }} \mathrm{NN}$ distance in the Al- $\mathrm{Cr}$ RDFs demonstrates that $\mathrm{Al}$ substitutes for $\mathrm{Cr}$ in the SRO domains leading to less retained $\mathrm{Cr}$. Unlike for $\mathrm{Al}-A l$ RDFs, the shift in $\mathrm{Cr}-\mathrm{Cr}$ RDFs with nucleation is gradual and is positive as $\mathrm{Cr}$ partitions to $\gamma$, where a slight negative correlation at $120 \mathrm{~s}$ may correspond to $\mathrm{Cr}$ ordering in the domains. A strong Al- $N i$ ordering tendency at the $1^{\text {st }} \mathrm{NN}$ distance at $120 \mathrm{~s}$ damps toward unity with aging, establishing that the cumulative $\mathrm{L}_{2}$-ordering (SRO and

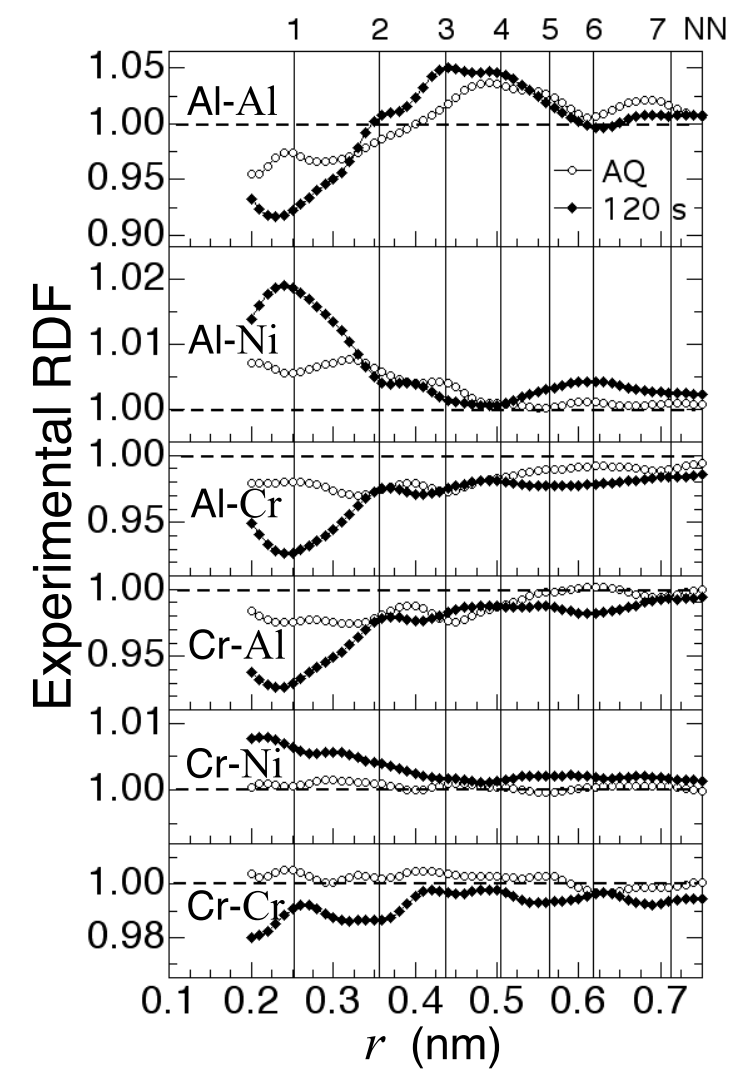

FIG. 3: Experimental RDF vs. radial distance, $r$, out to the $7^{\text {th }} \mathrm{NN}$ distance for as-quenched (AQ) Ni-5.2 Al-14.2 Cr at.\% and after aging for $120 \mathrm{~s}$ at $873 \mathrm{~K}$. The negative and positive correlations at the $1^{s t} \mathrm{NN}$ in the $\mathrm{Al}-\mathrm{Al}$ and $\mathrm{Al}-\mathrm{Ni}$ profiles establish the presence of $\mathrm{L}_{2}$-order.

LRO) in $\gamma+\gamma^{\prime}$ diminishes. Since $\gamma^{\prime}$-nucleation clearly leads to localized LRO development, the observed Al- $\mathrm{Ni}$ $\mathrm{RDF}$ damping corresponds to SRO diminishment in the $\gamma$-matrix with aging.

Lattice kinetic Monte Carlo (LKMC) simulations [9] of a nearly identical alloy, Ni-5.2 Al-14.8 $\mathrm{Cr}$ at.\%, aged at $873 \mathrm{~K}$, indicate that $\gamma$ '-phase separation occurs by nucleation and growth, which is preceded by $\mathrm{Ni}_{3} \mathrm{Cr}$-type $\left(\mathrm{DO}_{23}\right)$ SRO that develops, decays, and is followed by $\mathrm{L}_{2}$ SRO development. We do not observe this behavior experimentally, but find that the RDFs establish $\mathrm{L} 1_{2}$ SRO in the AQ state, which becomes stronger after 120 s, Fig. 3. In the AQ alloy, the distribution of $\mathrm{Cr}$ and $\mathrm{Ni}$ atoms relative to $\mathrm{Cr}$ atoms is initially random, hence $\mathrm{Ni}_{3} \mathrm{Cr}$ SRO does not precede the $\mathrm{L}_{2}$ SRO. The onset of Al-rich $\gamma^{\prime}$-phase separation (Fig. (4) occurs after $300 \mathrm{~s}$ of aging after SRO has occurred. A crucial difference between our experiments and the LKMC simulation [9] is the thermal history of the AQ state. In the LKMC simulation the initial state is a random distribution of atoms, which neglects the influence of a finite quench rate from $1123 \mathrm{~K}$, followed by up-quenching to $873 \mathrm{~K}$. The influence of the quench rate is twofold. Firstly, it leads to an 


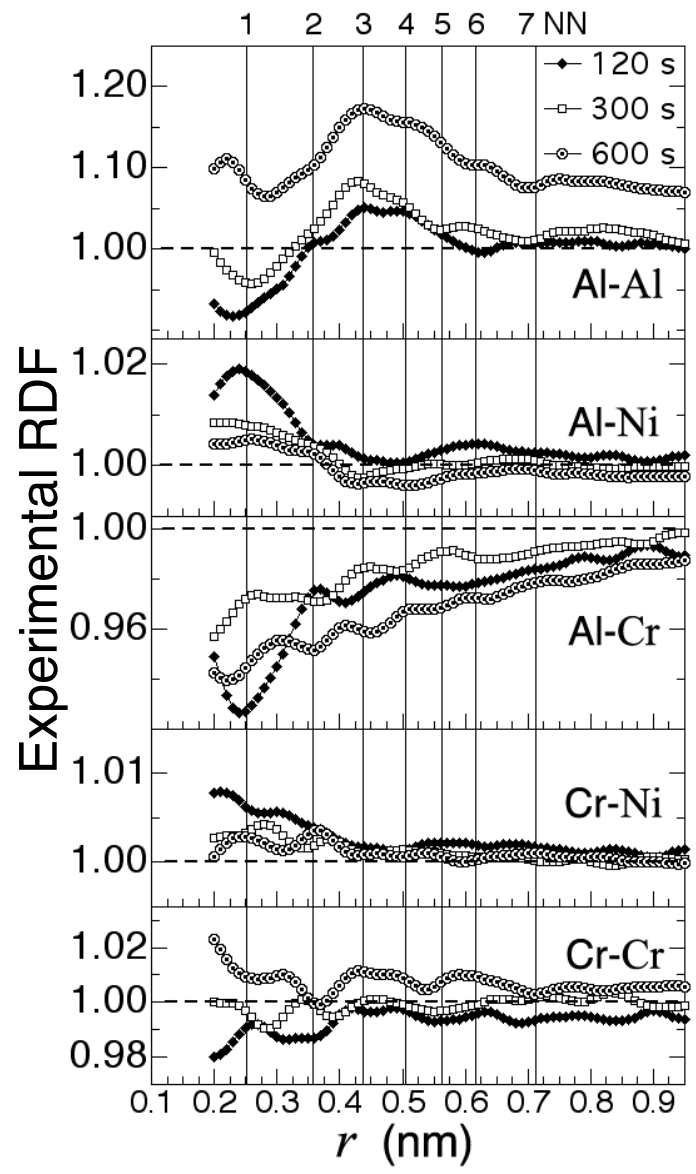

FIG. 4: Experimental RDF vs. radial distance, $r$, for Ni-5.2 Al-14.2 Cr at.\% aged for 120,300 , or $600 \mathrm{~s}$ at $873 \mathrm{~K}$. The overall $\mathrm{Al}-A l$ shift to higher RDF values after $300 \mathrm{~s}$ corresponds to the initiation of phase separation by $\gamma^{\prime}$-nucleation.

unknown value for the supersaturation of quenched-in vacancies. Secondly, phase separation may occur, particularly in alloys that are highly supersaturated and/or have a small interfacial free-energy, where the nucleation barriers are small and nucleation currents large [1, 2, 3]. For dislocation densities ranging between $10^{10}$ to $10^{12}$ $\mathrm{m}^{-2}$, the half-life time for the annihilation of excess vacancies at $873 \mathrm{~K}$, for diffusion-limited annihilation, is between 10118 and $107 \mathrm{~s}$, which are lower bounds since dislocation climb is assumed to be diffusion-limited [13], whereas it need not be 14]. This suggests that quenchedin excess vacancies may play a role in the early-stages of decomposition.

In summary, the chemical pathways leading to $\gamma^{\prime}$ nucleation at $873 \mathrm{~K}$ from a solutionized nondilute $\mathrm{Ni}$ $\mathrm{Al}-\mathrm{Cr}$ alloy are characterized in detail with isoconcentration surfaces and RDF (Eq. 1) analyses of direct-space APT images, Figs. 1-4. Our new approach offers the unique ability to characterize these pathways in nondilute multicomponent alloys, which are inherently complex, in particular when elemental diffusivities differ sig- nificantly. The application of an RDF to APT images provides a highly sensitive methodology to test homogeneity, which despite the APT's uncertainty in positioning absolutely atoms allows lattice-site ordering and clustering to be investigated, Fig. 2] An alloy's thermal history, in addition to quenched-in vacancies, influences the chemical pathways, which differ from predictions by LKMC simulations assuming a completely random solidsolution [9]. The finite quench-rate after solutionizing is sufficient to yield a random distribution of $\mathrm{Cr}$ atoms, Fig. 3. Quenching, however, produces a distribution of $\mathrm{Ni}_{3}\left(\mathrm{Al}_{x} \mathrm{Cr}_{1-x}\right)$ SRO $\left(\mathrm{L}_{2}\right)$ domains $(<R>\cong 0.6 \mathrm{~nm})$ that are congruently-ordered and $\mathrm{Cr}$-depleted relative to the solid solution. Initially, trapped $\mathrm{Cr}$ atoms show an antisite preference for the $\mathrm{Al}$ sublattice. With aging, as the $N_{v}$ of SRO domains increases and then $\gamma^{\prime}$-nuclei form, $\mathrm{Cr}$ is expelled and substituted for by Al. As highly localized LRO regions are established ( $\gamma^{\prime}$-nuclei), the SRO in the $\gamma$-matrix diminishes. A strong shift in $\mathrm{Al}-A l \mathrm{RDF}$ (Fig. (4) coincides with the detection of $\gamma^{\prime}$-nuclei (Fig. 1), thereby establishing that the onset of phase separation occurs between 300 and $600 \mathrm{~s}$ after SRO establishment. Between 600 and $900 \mathrm{~s}$, nuclei as small as $R=0.45 \mathrm{~nm}$ are detected, while the $N_{v}$ of $\gamma^{\prime}$-nuclei increases sharply as their $\langle R\rangle$ value remains constant $(0.75 \mathrm{~nm})$, Fig. [1]

This research is sponsored by the National Science Foundation, grant DMR-0241928; CKS received partial support from an NSF graduate fellowship. We thank Dr. K. E. Yoon for providing some APT data, and Dr. Z. Mao and Prof. M. Asta for helpful discussions.

* Electronic address: csudbrack@alumni.reed.edu

$\dagger$ Electronic address: d-seidman@northwestern.edu

[1] R. Wagner et al., Phase Transformations in Materials, edited by G. Kostorz (Wiley-VCH, Weinheim, 2001) p. 309.

[2] K. C. Russell, Adv. Coll. Int. Sci. 13, 205 (1980).

[3] F. Soisson and G. Martin, Phys. Rev. B 62, 203 (2000).

[4] H. I. Aaronson and F. K. LeGoues, Metall. Trans. A 23, 1915 (1992).

[5] M. J. Stowell, Mater. Sci. Tech. 18, 139 (2002).

[6] C. K. Sudbrack, Ph.D. thesis, Northwestern University (2004). http://arc.nucapt . northwestern. edu/refbase/show.php?record=

[7] O. C. Hellman, J. Blatz du Rivage, and D. N. Seidman, Ultramicro. 95, 199 (2003).

[8] E. A. Marquis et al., Phys. Rev. Let. 91, 036101 (2003).

[9] C. Pareige et al., Acta Mater. 47, 1889 (1999).

[10] O. C. Hellman et al., Microsc. Microanal. 6, 437 (2000).

[11] This APT volume contains 295,705 atoms, including trace amounts of $\mathrm{Ta}, \mathrm{Cr}$ and $\mathrm{B}$, where the measured composition of the $\mathrm{Ni}_{3} \mathrm{Al}$ lattice is $C_{N i}=73.93 \pm 0.16, C_{A l}=$ $25.29 \pm 0.16, C_{T a}=0.51 \pm 0.03$, and $C_{C r}=0.27 \pm 0.02$ at.\%, with $456 \mathrm{~B}$ atoms occupying interstitial sites.

[12] G. P. E. M. van Bakel, K. Hariharan and D. N. Seidman Appl. Surf. Sci. 90, 95 (1995). 
[13] D. N. Seidman and R. W. Balluffi, Phys. Stat. Sol. 17, 531 (1966).

[14] D. N. Seidman and R. W. Balluffi, in Lattice Defects and
Their Interactions, edited by R. R. Hasiguti (Gordon and Breach Science Publishers, New York, 1967) p. 911. 